tronischer-fussfessel-befassen/, abgerufen am 20. Februar 2012; kritisch zur VerfassungsmäBigkeit Pollähne 2011, S. $63 \mathrm{ff}$

58 Vgl. LT-Drucks. Mecklenburg-Vorpommern 5/4141; www.ostsee-zeitung.de/ index _ artikel _komplett.phtml? param $=$ news $\&$ id $=3067270$, abgerufen am 20. Februar 2012.

59 Staatsvertrag abgedruckt in LT-Drucks. BadenWürttemberg 15/1041.

60 www.welt.de/newsticker/dpa_nt/regioline_nt/ hamburgschleswigholstein_nt/article 1353 9876/Laender-kooperieren-bei-elektronischerFussfessel.html, abgerufen am 20. Februar 2012; LT-Drucks. Mecklenburg-Vorpommern $6 / 29$.

61 Im März 2011 mit polizeilicher Beobachtung; der Proband steht 2012 nicht mehr unter der elektronischen Aufenthaltsüberwachung; vgl. www.rp-online.de/panorama/deutschland/ju-
stiz/Erster-Sexualstraf-taeter-bekommt-elektronische-Fussfessel_aid_982841.html, abgerufen am 16.08.2011.

$62 \mathrm{Am}$ 28.06.2011 www.welt.de/regionales/ hamburg/article13500760/Erster-Haeftlingbekommt-elektro-nische-Fussfessel.html abgerufen am 16.08.2011.

$63 \mathrm{http}: / /$ www.neues-deutschland.de/artikel/21 9498.tragbares-gefaengnis.html, abgerufen am 20. Februar 2012; http://www.wetterauer-zeitung.de/Home/Kreis/Staedte-und-Gemeinden/ Bad-Vilbel/Artikel,-Die-Fussfessel-ist-keinAllheilmittel-_arid,316644_costart,2_regid,3_ puid,1_pageid,87.html, abgerufen am 20. Februar 2012.

64 Vgl. Mair 2005, S. 274: „Simply by institutionalizing pilot schemes and expanding its areas of application, electronic monitoring has mo- ved beyond any need for evidence to support it."

65 Royen 2012, S. 9.

66 Brauneisen 2011; BVerfG, 2 BvR 581/01 v. 10.11.2004.

67 So auch Pollähne 2011.

68 Harris et al. 2003, Nunes et al. 2002, Stadtland et al. 2006.

$69 \mathrm{Vgl}$. den Streit um die Aufgabe des Instituts für Sexualmedizin der Universität Kiel; mittlerweile scheint der Fortbestand des Instituts gesichert, wenngleich noch unklar zu sein scheint, ob die Patientenversorgung gewährleistet ist und die Präventionsprojekte fortbestehen werden; vgl. hierzu Deutschlandradio unter http://www. dradio.de/dlf/sendungen/campus/1685096/, abgerufen am 01.03.2012.

\title{
Zur prozeduralen Rechtfertigung von Whistleblowing in der Privatwirtschaft nach Schweizer (Straf-)Recht de lege ferenda
}

Andreas Eicker $^{7}$

\section{Einleitung}

In der Schweiz waren jüngst vor allem zwei Fälle zum Thema (externes) Whistleblowing in der öffentlichen Diskussion: der eine betrifft einen ehemaligen Julius-Bär-Banker, dem im Jahr 2011 erneut vorgeworfen wurde, steuerrechtlich relevante Kundendaten an Steuerbehörden, die Medien und an WikiLeaks weitergegeben zu haben. ${ }^{2}$ Der andere Fall betrifft einen EDV-Mitarbeiter der Privatbank Sarasin, der Hinweise darauf fand, dass ausgerechnet der Präsident der Schweizer Nationalbank als Hüter der Währung und Wechselkurse über ein Privatkonto Dollar ge- und verkauft und mit diesen Devisengeschäften ein sattes Plus erwirtschaftet haben soll. Diese Information fand ihren Weg zur rechtskonservativen Schweizerischen Volkspartei (SVP) und an die Öffentlichkeit; der Notenbankchef musste infolge dessen am 9. Januar 2012 per sofort zurücktreten. ${ }^{3}$ In Erinnerung sind ausserdem weitere Fälle, die ebenfalls ein erhöhtes mediales Interesse gefunden und zudem das Bundesgericht beschäftigt haben. Ein Fall, der am 12. Dezember 2011 entschieden wurde, betraf die Übergabe von internen Dokumenten durch zwei Mitarbeiterinnen des Sozialdepartements der Stadt Zürich an einen Zeitungsjournalisten ${ }^{4}$ und ein anderer
- etwas länger zurückliegender Fall - betraf eine Angestellte eines Pflegeheims, die ohne Wissen des Arbeitgebers nachts im Inneren des Heims einen Film gedreht hatte, und diesen dem westschweizerischen Fernsehen übergab, welches den Film in einer kritischen Sendung ausstrahlte. ${ }^{5}$

Das Thema Whistleblowing beschäftigt aber nicht nur immer wieder die Medien und die Gerichte, auch der Schweizer Gesetzgeber war und ist mit Massnahmen zum Schutz des Whistleblowers befasst. ${ }^{6}$ Seit dem 1. Januar 2011 besteht mit Art. 22a Bundespersonalgesetz (BPG) für Bundesangestellte eine ausdrückliche Regelung über das Melderecht und die Meldepflicht bei festgestellten Missständen. Ein Bericht der OECD, der im Januar 2012 veröffentlicht wurde, lobt diesen Schritt des Bundes, fordert aber besseren Schutz von Whistleblowern in der Privatwirtschaft. Diesbezüglich liegt ein Vorentwurf für eine Teilrevision des Obligationenrechts (OR) nach erfolgter Vernehmlassung nun wieder beim Bundesrat. ${ }^{7}$

Im rechtswissenschaftlichen Schrifttum der Schweiz ist die Thematik "Whistleblowing“ ebenfalls aktuell, wie mehrere Publikationen aus dem vergangenen Jahr zeigen. ${ }^{8}$ Nicht behandelt wurde sie bisher allerdings unter dem Aspekt der Prozeduralisierung des Strafrechts, weshalb dieser Bezugspunkt hier zum Gegenstand dieses Beitrags gemacht wird.

Whistleblowing als (straf-)rechtliches Problem

Wenn im Weiteren der Anglizismus „Whistleblowing " thematisch mit den Termini „prozedural“ bzw. „Prozeduralisierung“ verknüpft werden soll, dann lässt dies zunächst eine begriffliche Klärung ihrer jeweiligen Bedeutung erwarten. Die Prozeduralität des Strafrechts ${ }^{9}$ ist allerdings ein so „wenig bestimmter Begriff“ ${ }^{10}$, unter dem „viel Verschiedenes verstanden wird" ${ }^{\text {" } 11}$, dass er sich kaum im Rahmen einer Einleitung entfalten lässt, seine Bedeutung wird daher nachfolgend separat zu klären sein. Einfacher ist es mit dem Begriff Whistleblowing. Der Whistleblower kann ins Deutsche übersetzt auch als Hinweisgeber bzw. Anprangerer von Missständen bezeichnet werden, womit man allerdings das hinter dieser Begrifflichkeit stehende Phänomen nicht wirklich erfasst. Wird der Whistleblower als Denunziant gebrandmarkt, erhält der Terminus eine unnötig negative Wendung. Sachgerecht erscheint es daher, ihn als eine Person zu umschreiben, die wegen ihrer Stellung innerhalb eines Unternehmens, ei- 
ner Behörde oder einer anderen Organisation Informationen über rechtswidrige oder unethische (Geschäfts-)Praktiken dieser Organisation erlangt, und diese Kenntnisse an Dritte (z.B. die Medien) weitergibt. Dies mit der expliziten oder impliziten Aufforderung oder wenigstens Erwartung, dass gegen das als verwerflich empfundene Handeln eingeschritten wird. ${ }^{12}$

Dieses derart verstandene Offenlegen von illegalen oder unethischen Geschäftspraktiken seitens des Whistleblowers ${ }^{13}$ führt neben arbeits- und dienstrechtlichen Problemen auch zu strafrechtlichen Fragen. Denn wer z.B. Gesetzesverletzungen seines Arbeitgebers publik macht und damit zugleich ein Fabrikations- oder Geschäftsgeheimnis „verrät“ oder ein Amtsgeheimnis „offenbart“, erfüllt entweder den Straftatbestand von Art. 162 Abs. 1 oder von Art. 320 Ziff. $1 \mathrm{StGB}^{14}$. Zu erwähnen sind in diesem $\mathrm{Zu}$ sammenhang auch die Art. 273 StGB (wirtschaftlicher Nachrichtendienst) und Art. 6 UWG $^{15}$ (Verletzung von Fabrikations- und Geschäftsgeheimnissen). Für den Bereich der Finanzdienstleistungen ist die Geheimnisverletzung in Art. 47 BankG $^{16}$ und in Art. $43 \mathrm{BEHG}^{17}$ speziell geregelt. Aktuell sieht sich bspw. SVP-Nationalrat Blocher, der für sich in Anspruch nimmt, im eingangs erwähnten Fall Hildebrand (inzw. zurückgetretener Notenbankchef) als Whistleblower auf Missstände aufmerksam gemacht zu haben, mit dem Vorwurf der Verletzung des Bankgeheimnisses konfrontiert. ${ }^{18}$

Wenn nicht ohnehin gesetzlich geregelte Auskunfts- und Zeugnispflichten bestehen, ${ }^{19}$ wird in Fällen der Geheimnisoffenbarung die Frage aktuell, ob es Situationen gibt, in denen das Hinweisgeben strafrechtlich zu rechtfertigen ist. ${ }^{20}$ Für das Whistleblowing ausserhalb öffentlich-rechtlicher Arbeitsverhältnisse ${ }^{21}$, also im privaten Sektor, sieht der Vorentwurf zur Teilrevision des Obligationenrechts vor, im OR Voraussetzungen zu normieren, bei deren Erfülltsein der Arbeitnehmer nicht gegen seine Treuepflicht verstösst, wenn er Missstände in „seinem“ Unternehmen meldet oder sogar öffentlich macht. Wenn diese in Art. 321 $\mathrm{a}^{\text {bis }}$ OR des Vorentwurfs zur Änderung des Obligationenrechts vorgesehenen Anforderungen vom Arbeitnehmer erfüllt werden, wirken sie über Art. 14 StGB im Strafrecht als Rechtfertigung des Whistleblowings. ${ }^{22}$ Denn nach Art. 14 StGB ,verhält sich rechtmässig“, „wer handelt, wie es das Gesetz gebietet oder erlaubt, [...], auch wenn die Tat nach diesem [dem StGB] oder einem andern
Gesetz mit Strafe bedroht ist. " Dass dies in der Sache als ein prozeduraler Rechtfertigungsgrund verstanden werden kann, ist im Folgenden darzulegen.

\section{Prozeduralisierung und prozedurale Rationalität}

Während Stratenwerth - als prominentester Vertreter der Schweizer Strafrechtswissenschaft - Prozeduralisierung als „Fremdkörper“ im Strafrecht bezeichnet, ${ }^{23}$ exemplifiziert Hassemer vornehmlich am deutschen Recht, dass Prozeduralisierung im materiellen Strafrecht ein „passendes und legitimes Instrument“ sein kann. ${ }^{24}$ Dies vor allem dann, wenn es um die prozedurale Rechtfertigung von an sich strafbarem Verhalten geht. ${ }^{25}$ In diesem Sinne haben sich insbesondere auch Saliger ${ }^{26}$ und Popp ${ }^{27}$ mit dem „Konzept Prozeduralisierung “28 bzw. einem „prozeduralen Rechtfertigungskonzept “29 befasst. $^{30}$

Wenn aus den verschiedenen Prozeduralisierungstheorien und -modellen, die im Schrifttum vorgestellt werden, ein gemeinsamer Grundgedanke herausgefiltert wird, wie es der Verfasser dieses Beitrags in seiner Habilitationsschrift ${ }^{31}$ getan hat, dann sind prozedurale Regelungen im (Straf-)Recht solche, die das Recht darauf beschränken, angestrebte Ziele (z.B. Rechtsgüterschutz, Rechtfertigung von Verhalten) indirekt durch die Einrichtung und rechtliche Normierung von selbstregulatorischen Prozeduren zu erreichen. ${ }^{32}$ Anders gewendet bedeutet die Rechts-Prozeduralisierung einen partiellen „Rückzug des Rechts“ auf „prozedurale Programmierung “. ${ }^{33}$ Für die hier angesprochene Ebene der Rechtfertigungsgründe bezeichnet Prozeduralisierung mithin, so Saliger, „die von der Einhaltung spezifischer Verfahrensnormen34 abhängende Straflosigkeit rechtsgutsverletzender Handlungen." 35 Popp weist ergänzend darauf hin, dass der prozedurale Unrechtsausschluss grundsätzlich auch schon auf Tatbestandsebene erfolgen kann. ${ }^{36}$ Diese allgemeine Definition lässt allerdings ein extensives und ein restriktives Verständnis des Begriffs „Verfahren“ bzw. „Prozedur“ zu. Einerseits kann ganz generell die Einhaltung von $\mathrm{Zu}$ ständigkeits-, Formerfordernissen und anderen formellen Voraussetzungen gemeint sein; andererseits kommen Verfahrensregelungen in Betracht, die zusätzlich ein interpersonal-diskursives (kommunikatives) Element enthalten, wie bspw. ein Beratungsoder Zustimmungserfordernis. ${ }^{37}$ In der einschlägigen Literatur sind beide Lesarten gebräuchlich. ${ }^{38}$ Popp hält unter dem Stichwort „Prozeduralisierung“ fest, dass „dem Recht gemäss handelt [...], wer sein Handeln am Ausgang eines solchen Verfahrens orientiert.“ „Jede Entscheidung wird akzeptiert [und ist „nicht rechtwidrig"], solange und soweit sie auf einem konkreten Verfahren beruht. “39 Welches Verständnis der angesprochenen Lesarten „richtig“ ist, kann hier nicht entschieden werden. Hinzuweisen ist allein darauf, dass eine unter Einhaltung eines diskursiven Elements getroffene Entscheidung im Regelfall wohl eine höhere Legitimitätswirkung entfaltet, als eine solche, die lediglich unter Beachtung formeller Anforderungen zustande gekommen ist. ${ }^{40}$ Der Verfasser hat an anderer Stelle ${ }^{41}$ bereits darauf hingewiesen, dass die „Institutionalisierung von Kommunikation "42 i.w.S. die Richtigkeit bzw. Vernünftigkeit eines Ergebnisses zwar nicht garantieren, aber wenigstens begünstigen kann. Das diskursive Element ist mit anderen Worten eine die Plausibilität von Verfahrensentscheidungen unterstützende Bedingung, die das Hervorbringen von prozeduraler (Entscheidungs-) Rationalität ermöglicht und damit die Entscheidungsakzeptanz fördert. ${ }^{43}$

Dies vor allem deshalb, weil die Integration eines prozeduralen Elements ins Recht es ermöglicht, jene Voraussetzungen, die zur Rechtfertigung von an sich strafbarem Verhalten führen, bereits ex-ante festzustellen und den Handelnden in seiner Entscheidung zum Handeln nicht alleine zu lassen mit dem Risiko einer erst ex-post geführten strafrechtlichen und für ihn ggf. negativen Beurteilung dieser Voraussetzungen. ${ }^{44}$ Zwar hat es auch bei prozedural getroffenen Entscheidungen mit der „Vorabprüfung “ nicht sein Bewenden, vielmehr bleibt eine strafverfahrensrechtliche ex-post-Kontrolle der Einhaltung der prozeduralen Anforderungen möglich, ${ }^{45}$ doch gibt die Beachtung des rechtlich normierten Verfahrens dem Handelnden schon ex-ante die Gewissheit, dass er verfahrensmässig korrekt gehandelt hat, so dass im Rahmen einer ex-post-Beurteilung kaum zu erwarten ist, dass sein Handeln als rechtwidrig bewertet wird. Im Sinne von Rechtssicherheit geht es mithin um die Etablierung eines „präventiven Rechtstyps “46, mit dem einerseits das Interesse des Rechtsgutsträgers (z.B. Wahrung von Geschäftsgeheimnissen) geschützt und andererseits das Strafbarkeitsrisiko des Handelnden (z.B. des Whistleblowers), der (auch) ein berechtigtes Interesse verfolgt (z.B. Aufklärung von Missständen), minimiert werden kann. ${ }^{47}$ Jung spricht anschaulich davon, dass solche 
Prozeduren der „Austarierung [von] Interessen “ dienen. ${ }^{48}$ Mit Popp kann dies dahingehend umschrieben werden, dass vom Strafverfahren aus betrachtet der Verfahrensgegenstand gewissermassen vorgelagert wird auf eine zeitlich vorausgehende Prozedur. Dass das Interesse des Täters, schon vor der Tat Gewissheit über das Vorliegen von Rechtfertigungsvoraussetzungen zu haben, für das Strafrecht nicht untypisch ist, wird schon daran ersichtlich, dass Rechtfertigungsgründe neben objektiven Elementen stets auch ein subjektives Rechtfertigungselement voraussetzen. ${ }^{49}$

Mit Blick auf die Schweiz hat - soweit ersichtlich - zuletzt Geth den Gedanken der Rechts-Prozeduralisierung auf das materielle Strafrecht bezogen, wobei er es in $\mathrm{Zu}$ sammenhang gestellt hat mit den strafrechtlichen Problemen im Bereich der passiven Sterbehilfe und des zum 1. Januar 2013 neu in Kraft tretenden Erwachsenenschutzrechts. ${ }^{50}$ Damit hat er das Thema „Prozeduralisierung des Strafrechts" allerdings (auch wieder) auf einen Gegenstand angewendet, der - neben den strafrechtlichen Regelungen zum Schwangerschaftsabbruch - sicher am häufigsten als Beispiel für die Prozededuralisierung von Strafrecht herhalten muss. ${ }^{51}$ Dies gilt gleichfalls für den Verfasser dieses Beitrags, der das Konzept der Rechts-Prozeduralisierung aber auch für die schweizerischen Geldwäschereistrafnormen ${ }^{52}$ und jene zur Terrorismusbekämpfung ${ }^{53}$ untersucht hat. ${ }^{54}$ Dieser Befund hinterlässt ein gewisses Unbehagen, weil offensichtlich eine Diskrepanz besteht zwischen theoretisch-konstruktiven Arbeiten, die ein Bild vom prozeduralen Recht zu zeichnen suchen, und den nur sehr spärlich vorhandenen Belegen für die Existenz prozeduraler Regelungen im materiellen Strafrecht. Dies, obwohl in der Strafrechtswissenschaft die „wachsende Rolle prozeduraler Elemente in der Gestaltung und Handhabung des Strafrechts " ausdrücklich betont wird. ${ }^{55}$

Mit Rücksicht darauf wird im Folgenden gefragt, inwiefern die im Vorentwurf zur Teilrevision des Schweizer Obligationenrechts in Art. $321 \mathrm{a}^{\text {bis }}$ vorgesehenen Regelungen, die den "Schutz bei Meldung von Missständen am Arbeitsplatz" betreffen, einen Beitrag leisten zur Prozeduralisierung des materiellen Strafrechts.

Art. 14 StGB i.V.m. Art. 321a $\mathrm{a}^{\text {bis }}$ OR als prozeduraler Rechtfertigungsgrund

Wenn seitens eines Arbeitnehmers auf Missstände im Unternehmen aufmerksam ge- macht wird, indem z.B. Informationen an die Medien gegeben werden, dann kann ein solcher Hinweis grundsätzlich jeden Straftatbestand betreffen; meistens dürfte es sich allerdings um Wirtschaftskriminalität handeln. ${ }^{56}$ Ein solches Whistleblowing wird für den Arbeitnehmer selbst zum strafrechtlichen Problem, wenn die preisgegebene Tatsache vom Begriff des Geschäftsgeheimnisses erfasst wird. Denn nach Art. 162 Abs. 1 StGB macht sich strafbar, ,wer ein [...] Geschäftsgeheimnis, das er infolge einer gesetzlichen oder vertraglichen Pflicht bewahren sollte, verrät, [...]. “ Zum Begriff des Geschäftsgeheimnisses bemerkt das Bundesgericht, dass es sich dabei um Tatsachen handelt, die den kaufmännischen und/ oder betriebswirtschaftlichen Bereich eines Geschäfts oder Unternehmens betreffen und die weder offenkundig noch allgemein zugänglich sind und an deren Geheimhaltung der Geschäftsherr ein berechtigtes Interesse hat und die er geheim halten will. ${ }^{57}$ Unproblematisch vom Begriff des Geschäftsgeheimnisses erfasst werden insofern Tatsachen, wie z.B. Betriebsorganisation, Bezugsquellen, Absatzmöglichkeiten, Kundenkreis usw., die in keinerlei Beziehung zu einer im oder aus dem Unternehmen heraus begangenen Straftat stehen. An ihrer Geheimhaltung hat der Unternehmer zweifelsohne ein berechtigtes Interesse. Fraglich ist hingegen, ob die vom Whistleblower nach aussen getragene Information, dass im oder aus dem Unternehmen heraus eine Straftat begangen worden ist, eine vom Geheimnisbegriff des Art. 162 Abs. 1 StGB erfasste und damit geschützte Tatsache ist. Wichtig zu beachten ist nämlich, dass der objektive Tatbestand von Art. 162 Abs. 1 StGB infolge des soeben Gesagten dadurch eingeschränkt wird, dass nicht jedes Geschäftsgeheimnis geschützt wird, sondern nur ein solches, an dessen Schutz der Geheimnisherr und Arbeitgeber ein „berechtigtes“ Interesse hat. Trechsel z.B. plädiert dafür, dass es an einem schutzwürdigen Interesse fehlt, wenn im Unternehmen Waren gefälscht werden und mithin Straftaten nach Art. 155 Ziff. 1 StGB begangen wurden. ${ }^{58}$ Diese Sichtweise spricht dafür, dass der Whistleblower, der gegenüber Dritten äussert, im Unternehmen oder aus dem Unternehmen heraus seien bestimmte Delikte verübt worden, schon gar nicht den objektiven Tatbestand von Art. 162 Abs. 1 StGB erfüllt. Um dem Hinweis auf die Straftat eine gewisse Substanz $\mathrm{zu}$ verleihen und diesen überhaupt sinnvoll erscheinen zu lassen, wird es allerdings oft notwendig sein, auch Informationen aus dem Bereich des unzweifelhaft geschützten Geschäftsgeheimnisses (z.B. Kundenbeziehung) preiszugeben, womit der Whistleblower selbst in den Fokus der Strafverfolgung wegen Verletzung des Geschäftsgeheimnisses geraten kann. ${ }^{59}$ Im eingangs erwähnten Leitentscheid BGE 127 III 310 hat das Bundesgericht allerdings festgehalten, dass Arbeitnehmer bei Whistleblowing an die aus Art. 321a Abs. 4 OR folgende Pflicht gebunden sind. Diese Vorschrift bestimmt, dass ,der Arbeitnehmer [...] geheim zu haltende Tatsachen, wie namentlich Geschäftsgeheimnisse, von denen er im Dienst des Arbeitgebers Kenntnis erlangt, während des Arbeitsverhältnisses nicht verwerten oder anderen mitteilen" darf, so dass grundsätzlich die gesetzliche Pflicht besteht, (auch) Straftaten und andere Gesetzesverstösse des Arbeitgebers geheim zu halten. Dem stimmen Rieder und Imbach Haumüller ${ }^{60}$ zu. $^{61}$ Demnach erfüllt der Arbeitnehmer den objektiven Tatbestand von Art. 162 Abs. 1 StGB auch dann, wenn er strafbare Praktiken seines Arbeitgebers „verrät“. Dies wird einen potentiellen Whistleblower von der für ihn strafrechtlich riskanten Meldung von Missständen im Unternehmen abhalten. Auch wenn man die Auffassung vertritt, der Hinweis auf im oder aus dem Unternehmen heraus begangene Straftaten erfülle den objektiven Tatbestand von Art. 162 Abs. 1 StGB nicht, bleibt für den potentiellen Whistleblower doch zumindest immer die Rechtsunsicherheit, ob der Geheimnischarakter der Information tatsächlich nicht gegeben ist. Auch dies dürfte den potentiellen Hinweisgeber eher zurückhalten und dazu veranlassen, Stillschweigen zu bewahren. ${ }^{62}$

Abstrakt formuliert muss der potentielle Whistleblower also in einer Situation von Nichtwissen entscheiden, ${ }^{63}$ ob er seinem als berechtigt empfundenen Interesse folgt, auf Straftaten seines Arbeitgebers hinzuweisen. Dies, weil für ihn aus den genannten Gründen ex-ante nicht vorauszusehen ist, ob die von ihm preiszugebenden Informationen ein gesetzlich geschütztes „,berechtigtes Interesse" seines Arbeitgebers betreffen und ob ihm ggf. infolge seines Hinweises ex-post der Vorwurf gemacht wird, den Tatbestand von Art. 162 Abs. 1 StGB erfüllt zu haben.

Für den Bereich der passiven Sterbehilfe wird im Schweizer Schrifttum ${ }^{64}$ zum Teil dafür plädiert, dass in ähnlichen Konfliktsituationen ein Verfahren geschaffen werden müsse, welches es einerseits ermögliche, die Interessen des urteilsunfähigen Patienten bestmöglich $\mathrm{zu}$ schützen und andererseits 
das Strafbarkeitsrisiko des Arztes bzw. Sterbehelfers zu minimieren. Übertragen auf die Situation des Whistleblowings bedeutet dies, eine Prozedur vorzusehen, die ein berechtigtes Geheimhaltungsinteresse des Arbeitgebers berücksichtigt und es dem potentiellen Whistleblower erlaubt, die Informationen über Missstände im Unternehmen an Dritte preiszugeben bei minimalem Risiko, sich dadurch selbst strafbar zu machen. Geth hat zu Recht darauf hingewiesen, dass der Schweizer Gesetzgeber im Bereich passive Sterbehilfe mit dem neuen Erwachsenenschutzrecht (ab 01.01.2013) und der darin angelegten Stärkung der Kompetenzen der Erwachsenenschutzbehörde bereits in Richtung Prozeduralisierung des Rechts gehe. ${ }^{65}$ Aber nicht nur dort, auch im hier thematisierten Bereich Whistleblowing folgt der Vorentwurf zur Teilrevision des Obligationenrechts einem Weg, der als ein prozeduraler verstanden werden kann. Denn gemäss Art. 321a ${ }^{\text {bis }}$ OR de lege ferenda verstösst nicht gegen seine dem Arbeitgeber gegenüber bestehende Treuepflicht, wer - auch öffentlich, namentlich in den Medien - auf Missstände im Unternehmen hinweist, sofern er dabei das in dieser Vorschrift normierte Verfahren beachtet. ${ }^{66}$ Hält also der Arbeitnehmer, bevor er eventuell sogar die Medien informiert, das in Art. $321 \mathrm{a}^{\text {bis }}$ OR vorzusehende Prozedere ein, soll er im Idealfall bereits ex-ante wissen, dass ihm ex-post kein strafrechtsrelevanter Vorwurf wegen Verletzung von Art. 162 Abs. 1 StGB zu machen ist. Dies deshalb, weil ein verfahrensmässig korrektes Whistleblowing gemäss Art. $14 \mathrm{StGB}$ als prozeduraler Rechtfertigungsgrund im Strafrecht wirkt.

\section{Die prozeduralen Rechtfertigungs- voraussetzungen im Einzelnen}

Im Sinne des vorab zur Problematik des Entscheidens unter Bedingungen des Nichtwissens Gesagten hält auch der erläuternde Bericht zum Vorentwurf über die Teilrevision des Obligationenrechts fest, dass es „die Ungewissheit67 hinsichtlich der Berechtigung einer Meldung “ ist, die den Arbeitnehmer in den meisten Fällen veranlasst, Stillschweigen zu bewahren. Dies nicht zuletzt auch wegen der Angst vor den (straf-)rechtlichen Konsequenzen einer Meldung. Das Interesse an der Anzeige von Missständen erfordere deshalb „eine klare Festlegung auf Gesetzesebene". ${ }^{68}$ Im Sinne einer Prozeduralisierung des Rechts solle mithin künftig Art. $321 a^{\text {bis }}$ OR „das bei einer Meldung einzuschlagende Verfahren ${ }^{69}$ “ formalisieren.

\section{Grundsatz: interne Meldung}

Art. $321 \mathrm{a}^{\text {bis }}$ Abs. $1 \mathrm{OR}$ räumt zunächst der internen gegenüber der externen Meldung Priorität ein. Danach verstösst der Arbeitnehmer nicht gegen seine Treuepflicht, wenn er dem Arbeitgeber in Treu und Glauben Missstände meldet. Unter dem Begriff Arbeitgeber ist jede Stelle zu verstehen, die berechtigt ist, sich intern mit dem angezeigten Fall zu befassen. Hat der Arbeitgeber ausdrücklich eine bestimmte Prozedur für die Meldung von Missständen festgelegt, bestimmt ausschliesslich sie das massgebliche Vorgehen. ${ }^{70}$ Als Missstand gilt dabei jeder Sachverhalt, der im Widerspruch zu einer Verpflichtung des Unternehmens oder seiner Mitarbeiter steht. In erster Linie geht es dabei um strafbare Handlungen. ${ }^{71}$ Die Meldung muss „in Treu und Glauben“ erfolgen, d.h. der Arbeitnehmer muss zwar keine Gewissheit vom zurückliegenden oder noch andauernden Missstand haben, aber es müssen objektive Anhaltspunkte vorliegen, die ihn einen Missstand annehmen lassen. Wenn also ein späteres Strafverfahren zu der Erkenntnis führt, dass doch keine strafbare Handlung begangen wurde, macht dies das Whistleblowing des Arbeitnehmers nicht unberechtigt. $^{72}$

\section{Subsidiarität: extern-administrative Meldung}

Abs. 2 bestimmt, dass die Meldung an eine externe Behörde gegenüber dem unternehmensinternen Hinweis (vgl. Abs. 1) subsidiär ist. Dies ist Ausdruck des Verhältnismässigkeitsprinzips. Die externe Meldung ist auf jene Fälle beschränkt, in denen ein öffentliches Interesse berührt ist. Ein solches ist gegeben, wenn extra eine spezielle Behörde zur Entgegennahme von Meldungen über Missstände eingerichtet worden ist. Die Fälle, in denen eine externe Meldung erfolgen darf, sind in Abs. 2 festgelegt. Lit. a. geht davon aus, dass sich der Arbeitnehmer im Meldeverfahren prioritär an seinen Arbeitgeber wenden muss (vgl. dazu schon Abs. 1) und erst wenn dieser „nicht selber innert angemessener Frist wirksame Massnahmen [...] ergreift“, darf er die zuständige Behörde informieren. In den Bst. b. - d. sind jene Fälle aufgeführt, in denen es statthaft ist, direkt die zuständige Behörde einzuschalten. Dies ist der Fall, wenn gemäss lit. b. „anzunehmen ist, dass der Arbeitgeber keine wirksamen Massnahmen ergreifen wird“, weil er z.B. selbst in die Straftat verwickelt ist oder er schon früher bei anderen Meldungen nichts unternommen hat, oder nach lit. c. zu befürchten ist, dass „die Verfolgung von Taten $[. .$.$] vereitelt werden könnte, “$ indem bspw. Beweismittel vernichtet werden. Ausserdem darf die zuständige Behörde gemäss lit. d. direkt informiert werden, wenn „Gefahr im Verzug ist.“ Dies betrifft Krisensituationen, in denen z.B. Menschenleben gefährdet sind.

\section{Ultima ratio: extern-mediale Meldung}

Das prozedural ausgestaltete Melderecht sieht weiter in Abs. 3 vor, dass sich der Arbeitnehmer nur als ultima ratio an die Öffentlichkeit, namentlich an die Medien, wendet. Denn das Informieren der (Medien-)Öffentlichkeit ist aus der Perspektive des Arbeitgebers unter allen Umständen zu vermeiden, um einen möglichen Reputationsverlust zu verhindern. ${ }^{73}$ Wie der Arbeitgeber, muss auch die zuständige Behörde nicht innert angemessener Frist wirksame Massnahmen ergriffen haben, oder aufgrund besonderer Umstände muss anzunehmen sein, dass sie nichts unternehmen wird. Neben den Medien, an die sich der Arbeitnehmer als letztes Mittel wenden darf, erwähnt Abs. 3 auch noch „,interessierte Organisationen“. Darunter fallen z.B. Umweltschutz- und Konsumentenschutzorganisationen sowie Gewerkschaften. ${ }^{74}$

Wichtig zu beachten ist, dass die prozedurale Rechtfertigung aus Art. 14 StGB i.V.m. Art. 321 $\mathrm{a}^{\text {bis }}$ OR keine Anwendung findet auf Personen, die sich ans Berufsgeheimnis (vgl. Art. 321 f. StGB: Geistliche, Rechtsanwälte, Notare Ärzte usw.) zu halten haben oder für die spezialgesetzliche Regelungen gelten, vgl. Art. 321 $\mathrm{a}^{\text {bis }}$ Abs. 4 OR. Dieser Vorbehalt ist berechtigt, weil das Berufsgeheimnis, welches nur von bestimmten Berufen zu beachten ist, nicht mit der in einem Arbeitsverhältnis zu wahrenden Diskretion zu vergleichen ist. Vielmehr geht es um ein besonderes Auftrags- und Vertrauensverhältnis, welches, wenn es seinen Sinn und Zweck nicht verlieren soll, zu einer sehr restriktiven Handhabung des Melderechts führen muss. Es wird deshalb weiterhin Aufgabe der Praxis sein, in Form von allgemeinen Prinzipien einen Ausgleich zwischen dem Interesse an einer Meldung und dem Interesse an der Wahrung des Berufsgeheimnisses zu finden. ${ }^{75}$

\section{Bewertung: Prozeduralisierung durch regulierte Selbstregulierung notwendig}

Ziel der angesprochenen Gesetzesrevision ist es, den Arbeitnehmer als Whistleblower 
besser zu schützen, dies sowohl vor einer Vergeltungsmassnahme in Form einer ungerechtfertigten Kündigung als auch vor einer Strafverfolgung, insbesondere wegen Geheimnisverrats. Diese Zielsetzung ist zu begrüssen, doch wird die vorgeschlagene Revision dem Phänomen Whistleblowing nicht ausreichend gerecht. Im einschlägigen Schrifttum wird richtigerweise darauf hingewiesen, dass der Schutz von Hinweisgebern nur funktionieren kann - und damit ist das (notwendige) diskursive Element im Prozeduralisierungskonzept gemeint, „wenn in einem Unternehmen entsprechende Kommunikationsstrukturen vorhanden sind, die ein vertrauliches - und damit geschütztes - Whistleblowing tatsächlich zulassen.“76 Das im Vorentwurf vorgesehene Verfahren soll sicherstellen, dass Meldungen zuerst intern und nur in Ausnahmefällen extern (als ultima ratio gegenüber den Medien) erfolgen, um sowohl dem Unternehmensinteresse (z.B. Vermeidung negativer Presse, Reputationsverlust) als auch dem des Arbeitnehmers (Meldung von Missständen) Rechnung zu tragen. Abgesehen davon, dass das vorgesehene Kaskadenprinzip (zu) viele unbestimmte und damit auslegungsbedürftige Rechtsbegriffe enthält ${ }^{77}$ (z.B. „öffentliches Interesse“, „Treu und Glauben $\left.{ }^{\text {( }}\right)^{78}$, sagt es dem potentiellen Whistleblower nicht klar genug, an wen er sich intern oder extern wenden darf. Denn nach dem dargestellten Modell ist der Arbeitnehmer (vor Strafverfolgung) grundsätzlich nur geschützt, wenn er der richtigen Stelle Meldung macht. Nur wenn er diese kennt, kann er auch beurteilen, ob diese aufgrund seines Hinweises etwas unternimmt und er sich, falls dies nicht der Fall ist, auf die nächste Verfahrensstufe im Kaskadensystem begeben darf (zunächst externe Behörde, dann Öffentlichkeit). Der im Vorentwurf angelegte prozedurale Ansatz zum Schutz des Whistleblowers ist daher, worauf Portmann hinweist, um ein selbstregulatorisches leicht verständliches Meldeverfahren auf Unternehmensebene zu ergänzen, welches (auch) eine entsprechende Kommunikation gegenüber den Arbeitnehmern beinhaltet. Diese müssen konkret wissen, wie das Verfahren abläuft. ${ }^{79}$ Deshalb wird vorgeschlagen, die Einrichtung eines solchen internen Meldeverfahrens gesetzlich vorzuschreiben. ${ }^{80} \mathrm{Wie}$ dieses dann genau auszusehen hat, stünde im Ermessen des Arbeitgebers. ${ }^{81}$ Der Gesetzgeber würde mit anderen Worten, worauf Kälin und Kirchhoff hinweisen, nur die Handlungsleitplanken für den Arbeitgeber setzen, letzterem aber keine weiteren Vor- gaben machen; dies entspricht dem Konzept von regulierter Selbstregulierung als ein Wesensmerkmal prozeduralen Rechts. ${ }^{82}$ Ein Ansatz dafür - aber auch nicht mehr - ist im Schweizer Strafrecht bereits vorhanden. Denn nach Art. 102 Abs. 2 StGB kann ein Unternehmen strafrechtlich zur Verantwortung gezogen werden, wenn es nicht alle erforderlichen und zumutbaren organisatorischen Vorkehrungen trifft, um bestimmte (Katalog-)Straftaten zu verhindern. Diese Vorschrift stellt implizit die Pflicht auf, solche Straftaten durch entsprechende organisatorische Massnahmen - ggf. Verfahren - zu verhüten. Daraus liesse sich auch die Pflicht zur Einrichtung einer entsprechenden Meldestelle und einer an die Mitarbeiter kommunizierten internen (selbst regulierten) Meldeprozedur ableiten. ${ }^{83}$ In Erweiterung dieses Gedankens wird im aktuellen Schrifttum vorgeschlagen, in Art. 301 StPO (Anzeigerecht) eine Bestimmung aufzunehmen, mit der Whistleblowern Immunität vor strafrechtlicher Verfolgung zugesichert wird, sofern sie das zum fraglichen Zeitpunkt geltende Meldeverfahren eingehalten haben. ${ }^{84}$ Damit würde die materiellrechtliche prozedurale Rechtfertigung noch zusätzlich formellrechtlich abgesichert.

\section{Fußnoten}

1 Der Verfasser ist Ordinarius für Strafrecht und Strafprozessrecht an der Universität Luzern. Für die Überarbeitung des Manuskripts in formeller Hinsicht danke ich meiner Assistentin Frau BLaw Julia Lehner.

2 Vgl. z.B. Whistleblower Elmer raus aus U-Haft, Handelszeitung, 15. August 2011, <http:// www.handelszeitung.ch/unternehmen/whistleblower-elmer-raus-aus-u-haft> (besucht am: 14. März 2012) sowie „Jetzt beginnt die Ära der Whistleblower", Tagesanzeiger, 9. Januar 2012, <http://www.tagesanzeiger.ch/wirtschaft/ agenturen-ticker/Jetzt-beginnt-die-Aera-derWhistleblower/story/23432257> (besucht am. 14. März 2012).

3 Vgl. z.B. Auf schmalem Grat - Ein sogenannter Whistleblower brachte den Schweizer Notenbankchef Philipp Hildebrand zu Fall. Sind solche Enthüller Helden oder Verräter?, Der Spiegel 3/2012, S. $68 \mathrm{ff}$.

4 BGE 6B_305/2011 vom 12. Dezember 2011.

5 BGE 127 III 310 ff. vom 30. März 2001.

6 Vgl. dazu Bundesamt für Justiz, Vorentwurf zur Teilrevision des Obligationenrechts (Schutz bei Meldung von Missständen am Arbeitsplatz), <http://www.ejpd.admin.ch/content/dam/data/ wirtschaft/gesetzgebung/whistleblowing/entwd.pdf> (besucht am: 15. März 2012), der zurückgeht auf eine Motion von Nationalrat Remo Gysin mit dem Titel „Gesetzlicher Schutz für Hinweisgeber von Korruption" (Motion 03.3212 vom 7. Mai 2003) sowie neu Art. 22a des Bundespersonalgesetzes (BPG).

$7 \mathrm{Vgl}$. Mehr Schutz für Whistleblower, Informationsplattform humanrights.ch, 23. Januar 2012, <http://www.humanrights.ch/de/Schweiz/Inneres/Person/Verschiedenes/idart_224-content. html> (besucht am: 14. März 2012).
8 Vgl. z.B. Imbach Haumüller, Whistleblowing in der Schweiz und im internationalen Vergleich - ein Bestandteil einer effektiven internen Kontrolle?, Zürich 2011; von Kaenel (Hrsg.), Beiträge zum Whistleblowing, Bern 2012; ders., Whistleblowing, in: SJZ 103/2007, S. 309 ff. (im Folgenden: von Kaenel, SJZ 103/2007); Portmann, Gesetzliche Regelung des Whistleblowing in der Schweiz - Überflüssig, nützlich oder notwendig?, in: AJP/PJA 8/2010, S. 987 ff.; Rieder, Schutz für Whistleblower - Kommentar zum Gesetztesentwurf, in: Jusletter 20. April 2009; ders., Whistleblowing als Menschenrecht, in: Jusletter 28. November 2011; Kälin/Kirchhoff, Whistleblowing - Eine Anleitung, in: Jusletter 20. Juni 2011.

9 Vgl. dazu ausführlich Eicker, Die Prozeduralisierung des Strafrechts, Zur Entstehung, Bedeutung und Zukunft eines Paradigmenwechsels, Bern 2010 (im Folgenden: Eicker, Prozeduralisierung des Strafrechts) sowie Eicker, AntiTerrorismus-Gesetzgebung im Lichte der Prozeduralisierung des Strafrechts, in: Juchli/ Würmli (Hrsg.), Auswirkungen des Terrorismus auf Recht, Wirtschaft und Gesellschaft, Bern 2006, S. 197 ff. (im Folgenden: Eicker, Anti-Terrorismus-Gesetzgebung).

10 Stratenwerth, Prozedurale Regelungen im Strafrecht, in: Herzog/Neumann (Hrsg.), Festschrift für Winfried Hassemer zum 70. Geburtstag am 17. Februar 2010, Heidelberg u.a. 2010, S. 639 ff.

11 Eicker, Rezension zu Christopher Geth, «Passive Sterbehilfe» (und Prozeduralisierung), in: ZStrR 129/2011, S. 225 ff. (im Folgenden: Eicker, ZStrR 129/2011), S. 227.

12 von Kaenel, SJZ 103/2007, S. 309.

13 Kälin/Kirchhoff, a.a.O., Rz. 1.

14 Schweizerisches Strafgesetzbuch vom 21. Dezember 1937 (SR 311). Gesetzesangaben mit der Bezeichnung StGB beziehen sich im Folgenden auf dieses Gesetz.

15 Bundesgesetz vom 19. Dezember 1986 gegen den unlauteren Wettbewerb (SR 241). Gesetzesangaben mit der Bezeichnung UWG beziehen sich im Folgenden auf dieses Gesetz.

16 Bundesgesetz vom 8. November 1934 über die Banken und Sparkassen (SR 952.0).

17 Bundesgesetz vom 24. März 1995 über die Börsen und den Effektenhandel (SR 954.1).

18 Vgl. dazu Eicker, Neue Luzerner Zeitung vom 22.03.2012, S. 5.

19 Vgl. dazu im Einzelnen Erläuternder Bericht Teilrevision OR, S. 9 f.

20 Erläuternder Bericht Teilrevision OR, S. 9.

21 Für die öffentlichen Angestellten des Bundes vgl. Art. 22a Bundespersonalgesetz (BPG).

22 Erläuternder Bericht Teilrevision OR, S. 25.

23 Stratenwerth, a.a.O., S. 646.

24 Hassemer, Prozeduralisierung, Wahrheit und Gerechtigkeit. Eine Skizze, in: Pieth/Seelmann (Hrsg.), Prozessuales Denken als Innovationsanreiz für das materielle Strafrecht, Kolloquium zum 70. Geburtstag vom Detlef Krauss, Basel 2006, S. 9 ff., 12.

25 Hassemer, Prozedurale Rechtfertigung, in: Däubler-Gmelin/Kinkel/Meyer/Simon (Hrsg.), Gegenrede, Festschrift für Ernst Gottfried Mahrenholz, Baden-Baden 1994, S. 731.

26 Saliger, Prozedurale Rechtfertigung im Strafrecht, in Herzog/Neumann (Hrsg.), Festschrift für Winfried Hassemer zum 70. Geburtstag am 17. Februar 2010, Heidelberg u.a. 2010, S. 599 ff.

27 Popp, Patientenverfügung, mutmassliche Einwilligung und prozedurale Rechtfertigung, in: ZStW 118/2006, S. $639 \mathrm{ff}$.

28 Popp, a.a.O., S. 664 ff.

29 Saliger, a.a.O., S. 599 f.

$30 \mathrm{Vgl}$. zum Gedanken des ,prozeduralisierten Wirtschaftsstrafrechts“ auch Schneider, NK $1 / 2012$. 
31 Eicker, Prozeduralisierung des Strafrechts.

32 Eicker, Prozeduralisierung des Strafrechts, S. 121; vgl. auch Jung, ZStrR 130/2012, S. 39, 45.

33 Schulz/Held, Regulierte Selbstregulierung als Form modernen Regierens, in: Arbeitspapiere des Hans-Bredow-Instituts Nr. 8, 2001, S. A-9.

34 Hervorhebung hier durch den Verfasser.

35 Saliger, a.a.O., S. $601 \mathrm{f}$.

36 Popp, a.a.O., S. 664.

37 Saliger, a.a.O., S. 602.

38 Vgl. dazu die Darstellung bei Saliger, a.a.O., S. $602 \mathrm{f}$.

39 Popp, a.a.O., S. 665.

40 Saliger, a.a.O., S. 603.

$41 \mathrm{Vgl}$. Eicker, Prozeduralisierung des Strafrechts.

42 Eicker, ZStrR 129/2011, S. 227.

43 Eicker, Prozeduralisierung des Strafrechts, S. $131 \mathrm{ff}$, insb. S. $143 \mathrm{ff}$.

44 Eicker, ZStrR 129/2011, S. 228.

45 Saliger, a.a.O., S. 610

46 Saliger, a.a.O., S. 610

47 Eicker, ZStrR 129/2011, S. 227.

48 Jung, a.a.O., S. 45.

49 Popp, a.a.O., S. 667

50 Vgl. Geth, Passive Sterbehilfe, Basel 2010, S. 91 sowie S. 136 ff.; vgl. dazu auch Eicker, ZStrR 129/2011, S. 225 ff. sowie - ohne speziell auf das Schweizer Strafrecht zu fokussieren; Arzt, Patientenverfügung: Rechtsverlust durch Verfahren, in: Dannann/Grunsky/Pfeiffer (Hrsg.), Gedächtnisschrift für Manfred Wolf, München 2011, S. $609 \mathrm{ff}$.

51 Vgl. dazu die zahlreichen Nachweise bei Eicker, Prozeduralisierung des Strafrechts, S. 206 ff.

52 Vgl. Eicker, Prozeduralisierung des Strafrechts, S. $168 \mathrm{ff}$.

53 Vgl. Eicker, Anti-Terrorismus-Gesetzgebung.
54 Vgl. dazu Eicker, Prozeduralisierung des Strafrechts, S. $206 \mathrm{ff}$.

55 Eser, Sanktionierung und Rechtfertigung durch Verfahren. Eine Problemskizze, in: KritV, Sonderheft 2000, Winfried Hassemer zum 60. Geburtstag, S. $43 \mathrm{ff}$.

56 Rieder, Jusletter 20. April 2009, Rz. 1.

57 Vgl. z.B. BGE 80 IV 22 ff. (27), E. 2a; BGE 103 IV 283 ff. (284), E. 2b sowie Stratenwerth, Schweizerisches Strafrecht, Besonderer Teil I: Straftaten gegen Individualinteressen, 7. Aufl., Bern 2010, S. 511 Rz. 3.

58 Trechsel et al., Schweizerisches Strafgesetzbuch, Praxiskommentar, Zürich/St. Gallen 2008, Art. 162 Rz. 2.

59 von Kaenel, SJZ 103/2007, S. 312.

60 Rieder, Jusletter 28. November 2011, Rz. 15 unter Hinweis auf BGE 127 III 310 ff. (315), E. 5a.; Imbach Haumüller, a.a.O., Rz. 78, die diese Sichtweise als die in der (Schweizer) Lehre allgemein vertretene Auffassung bezeichnet.

61 Vgl. ebenfalls den Erläuternden Bericht Teilrevision OR, S. 8

62 Kälin/Kirchhoff, a.a.O., Rz. 5; von Kaenel, SJZ 103/2007, S. 312

63 Kälin/Kirchhoff, a.a.O., Rz. 5.

64 Geth, a.a.O., S. 91; Eicker, ZStrR 129/2011, S. 227.

65 Geth, a.a.O., S. 91.

66 Kälin/Kirchhoff, a.a.O., Rz. 7, 9

67 Hervorhebung hier durch den Verfasser.

68 Erläuternder Bericht Teilrevision OR, S. 16

69 Hervorhebung hier durch den Verfasser.

70 Kälin/Kirchhoff, a.a.O., Rz. 15 f.

71 Imbach Haumüller, a.a.O., Rz. 370; Kälin/ Kirchhoff, a.a.O., Rz. 12, 13.

72 Erläuternder Bericht Teilrevision OR, S. 38 f.
73 Kälin/Kirchhoff, a.a.O., Rz. 19.

74 Erläuternder Bericht Teilrevision OR, S. $41 \mathrm{f}$

75 Erläuternder Bericht Teilrevision OR, S. 26.

76 Imbach Haumüller, a.a.O., Rz. 499; Rieder, Jusletter 20. April 2009, Rz. 4.

77 Vgl. dazu ausführlich Portmann, a.a.O., S. 990 ff. sowie Eidgenössisches Justiz- und Polizeidepartement (EJPD), Medienmitteilung vom 17. Dezember 2009, Besserer Schutz von Whistleblowern mehrheitlich befürwortet - Bundesrat nimmt Vernehmlassungsergebnisse zur Kenntnis (im Folgenden: Medienmitteilung des EJPD), S. 7 f., <http://www.ejpd.admin.ch/ejpd/ de/home/dokumentation/mi/2009/2009-12-17. html> (besucht am: 15. März 2012) und Imbach Haumüller, a.a.O., Rz. 382.

78 Imbach Haumüller, a.a.O., Rz. 372; Kälin/ Kirchhoff, a.a.O., Rz. 17.

79 Portmann, a.a.O., S. 995; vgl. in diesem Sinne auch die Medienmitteilung des EJPD, S. 8 sowie Imbach Haumüller, a.a.O., Rz. 373, 499.

80 Portmann, a.a.O., S. 995; Rieder, Jusletter 20. April 2009, Rz. 9; zweifelnd Imbach Haumüller, a.a.O., Rz. 407.

81 Rieder, Jusletter, 20. April 2009, Rz. 9.

82 Kälin/Kirchhoff, a.a.O., Rz. 27; vgl. dazu genauer Eicker, Prozeduralisierung des Strafrechts, S. $118 \mathrm{ff}$. m.w.N.

83 Imbach Haumüller, a.a.O., Rz. 477; Forster, Die strafrechtliche Verantwortlichkeit des Unternehmens nach Art. 102 StGB, Bern 2006, S. 237; Portmann, a.a.O., S. 995; a.A. Gfeller, in Niggli/Wiprächtiger (Hrsg.), Basler Kommentar, Strafrecht I, 2. Aufl., Basel 2007, Art. 102 Rz. 305.

84 Imbach Haumüller, a.a.O., Rz. 403.

\section{Restorative Justice und Täter-Opfer-Ausgleich in Deutschland}

\section{- zwischen lästiger Pflicht und sozialintegrativem Potential}

Frank Winter, Eduard Matt

Der Beitrag zeigt die Möglichkeiten von Konfliktschlichtung und Tatfolgenausgleich als Maßnahmen einer restorative justice und diskutiert, wie unsere an rechtsstaatlichen Werten ausgerichtete Gemeinschaft (community1) mit Taten, Tätern, Opfern und einschneidenden, schlimmstenfalls traumatisierenden Situationen human umgehen könnte. Im Focus stehen die Idee einer wiederherstellenden „heilenden“ Praxis des zur strafenden Sanktion alternativen Umgangs mit Straftaten und die Frage, welche Hinderungsgründe derzeit noch immer bestehen, solche Praxis in Deutschland in größerem Umfang zur Anwendung zu bringen.

Grundlage bestehender restorative justiceVerfahren ist die Perspektive der Restitu- tion, des anhaltenden und wiedergutmachenden Ausgleichs zwischen Täter, Opfer und Gemeinschaft. Explizite Zielsetzung der Verfahren ist die soziale Reintegration von Geschädigten und Beschuldigten. Notwendige Elemente solcher Reintegration sind für die Beteiligten die Auseinandersetzung mit dem Tatgeschehen und den Tatfolgen. Diese Auseinandersetzung geschieht für die Beteiligten freiwillig und aus der jeweils individuellen Perspektive. Sie geschieht durch Perspektivenverschränkung, also durch den Austausch individueller Sichtweisen. Für das Opfer gilt es, die mit der Tat einhergehenden Erfahrungen von Kontrollverlust, Verlust an Sinngebung und Verlust des Glaubens an eine geordnete Welt zu bewältigen (Gehm 1998). Für den Täter steht am
Ende die Verantwortungsübernahme für das Tatgeschehen. Diese impliziert die Erbringung von Leistungen einer angemessenen Wiedergutmachung gegenüber dem Opfer. Ist kein personifiziertes Opfer vorhanden, wird die Wiedergutmachungsleistung gegenüber der Gemeinschaft z.B. durch die Ableistung einer gemeinnützigen Arbeit erbracht.

Die Gemeinschaft - wenn sie nicht selbst Opfer wurde - muss einerseits den Prozess der Herstellung des sozialen Friedens zwischen Opfer und Täter begleiten, andererseits muss sie beiden einen haltenden Rahmen bieten. Die Wiedereingliederung der Opfer und derjenigen Täter, die ihre Schäden wieder gutgemacht haben, ist durch die Gemeinschaft zu gewährleisten. Die grund- 